Internist 2013 · 54:1011-1015

DOI 10.1007/s00108-013-3327-3

Online publiziert: 10. Juli 2013

(c) Springer-Verlag Berlin Heidelberg 2013

\section{Rubrikherausgeber}

K. Werdan, Halle (Saale)

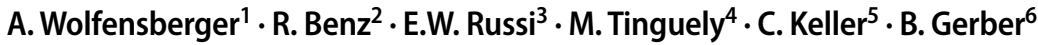 \\ ${ }^{1}$ Klinik für Infektionskrankheiten und Spitalhygiene, UniversitätsSpital Zürich \\ ${ }^{2}$ Klinik für Innere Medizin, Hämatologie, Kantonsspital Münsterlingen \\ ${ }^{3}$ Klinik für Pneumologie, UniversitätsSpital Zürich \\ ${ }^{4}$ Institut für Klinische Pathologie, UniversitätsSpital Zürich \\ ${ }^{5}$ Internistische Hausarztpraxis, Brugg \\ ${ }^{6}$ Klinik für Hämatologie, UniversitätsSpital Zürich
}

\title{
Husten, Atemnot und B-Symptome bei einer 40-jährigen Frau
}

\section{Anamnese}

Im Juli 2004 stellte sich eine 42 -jährige Patientin mit seit mehr als 2 Jahren bestehendem Husten, Dyspnoe, Adynamie, Myalgie und Nachtschweiß in unserem Zentrumsspital zur erneuten Abklärung vor. Zuvor waren eine interstitielle Pneumopathie mit restriktiver Ventilationsstörung und eine Einschränkung der CO-Diffusionskapazität (60\%) diagnostiziert worden. Eine thorakoskopische Lungenbiopsie im April 2002 hatte eine granulomatöse Entzündung mit „atypischen lymphoplasmazellulären Infiltraten“ ergeben. Bei Verdacht auf eine atypische Sarkoidose war im Anschluss an die Biopsie eine mehrmonatige Therapie mit Steroiden und Methotrexat eingeleitet worden, woraufhin es nicht zu einer klinischen Besserung kam. Die medizinische Vorgeschichte der Patientin war ansonsten unauffällig, insbesondere bestanden keine Allergien und keine Exposition potenzieller Noxen.

\section{Klinischer Befund}

Die 42-jährige Patientin war bei unserer Erstkonsultation in einem guten Allgemeinzustand, schlank (Body-Mass-Index: $23 \mathrm{~kg} / \mathrm{m}^{2}$ ) und afebril. Die Atemfrequenz betrug 20/min, die Herzfrequenz 76/min, der Puls war regelmäßig. Sowohl die Herz- als auch die Lungenauskulta- tion waren unauffällig. Es fand sich keine Lymphadenopathie, auch die Milz war nicht tastbar.

\section{Diagnostik}

\section{Computertomographie des Thorax und Abdomens}

In der Computertomographie fanden sich im Lungenparenchym disseminierte multiple noduläre Infiltrate, teilweise mit umgebenden Milchglasopazitäten, v. a. auch subpleural gelegen, tubuläre Traktionsbronchiektasen in beiden Unterlappen und Lymphknotenvergrößerungen hilär beidseits, paratracheal, infrakarinär und aortopulmonal (• Abb. 1).

\section{Labor}

Die Laboruntersuchung erbrachte folgende Ergebnisse: Hämoglobin 98 g/l (117$153 \mathrm{~g} / \mathrm{l})$; mittleres Erythrozytenvolumen (MCV) 83,3 fl (80-100 fl); mittlere Hämoglobinkonzentration in Erythrozyten (MCHC) 31,2 g/dl (31-36 g/dl); Thrombozyten $600 \bullet 10^{9} / 1\left(143-300 \bullet 10^{9} / 1\right)$; Leukozyten $9,38 \cdot 10^{9} / 1\left(3,0-9,6 \bullet 10^{9} / 1\right)$; normales automatisches Differenzialblutbild. C-reaktives Protein (CRP) $167 \mathrm{mg} / \mathrm{l}(<5 \mathrm{mg} / \mathrm{l})$; Laktatdehydrogenase, Kreatinin, alkalische Phosphatase, Aspartat-Aminotransferase (ASAT) und Alanin-Aminotransferase (ALAT) normwertig

\section{Videomediastinoskopie mit Lymphknotenbiopsie und thorakoskopische Lungenbiopsie aus dem linken Oberlappen}

Durch einen Schnitt am Jugulum und nach stumpfem Tunnelieren an der Vorderseite der Trachea wurde ein Mediastinoskop vorgeschoben. Daraufhin erfolgten die Entfernung eines großen Lymphknotens in toto und Biopsien aus stark veränderten und vergrößerten Lymphknoten. Nach Umlagerung der Patientin in Rechtsseitenlage wurde über eine Hautinzision in der linken vorderen Axillarlinie das Thorakoskop eingebracht. Die Oberfläche der Lunge war makroskopisch deutlich verändert. Der am deutlichsten veränderte Bezirk an der Lingulaspitze wurde parenchymsparend mit dem Endostapler reseziert.

\section{Histologie des Wedge-Lungenresektats}

Es fand sich eine gesteigerte, gemischtzellige lymphatische Proliferation ohne molekulargenetischen Nachweis einer monoklonalen B- oder T-Zell-Proliferation (Polymerase-Kettenreaktion und Southern Blot des IGH-Genlokus und SouthernBlot des TCR $\beta$-Genlokus). 

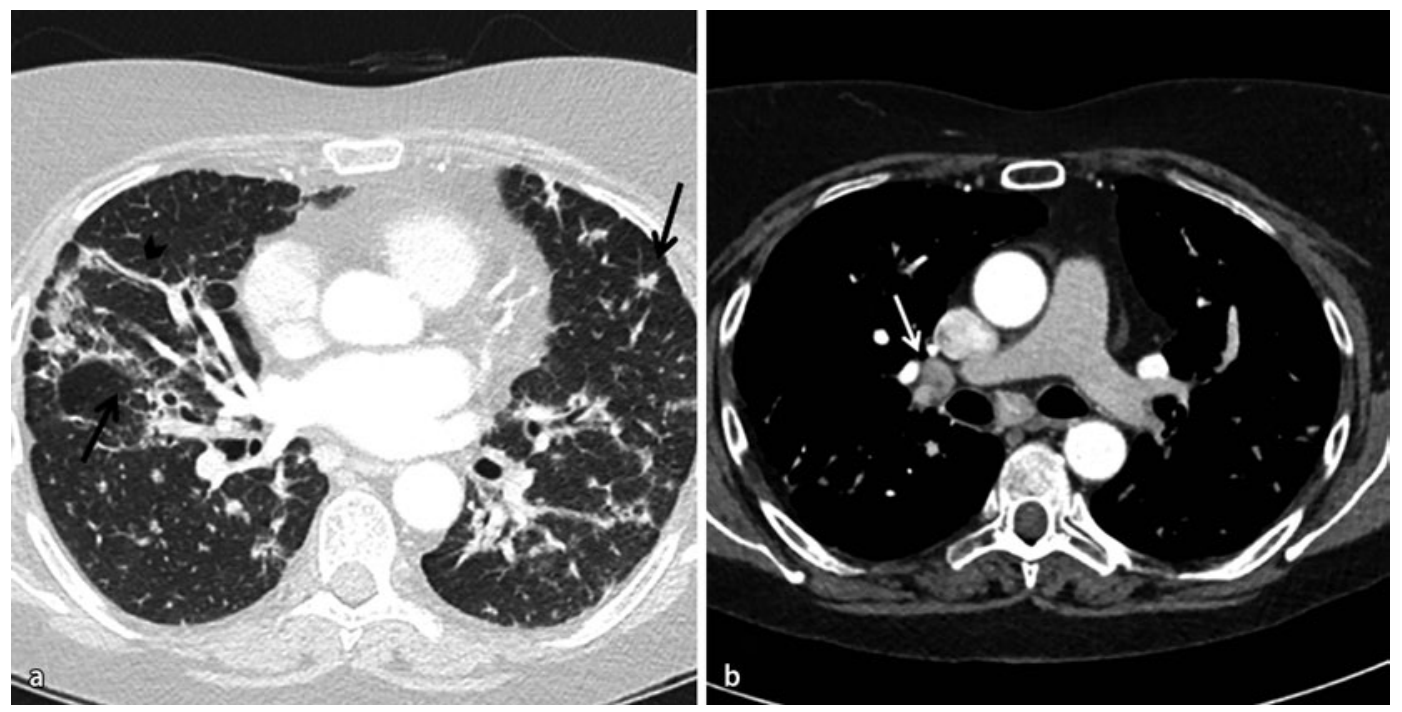

Abb. $1<$ a Lungenparenchym mit multiplen nodulären Infiltraten, teils mit umgebenden Milchglasopazitäten, v. a. auch subpleural gelegen (Pfeile). Tubuläre Traktionsbronchiektasen (Pfeilkopf). b Lymphknotenvergrößerung hilär beidseits, paratracheal, infrakarinär und aortopulmonal (Pfeil)
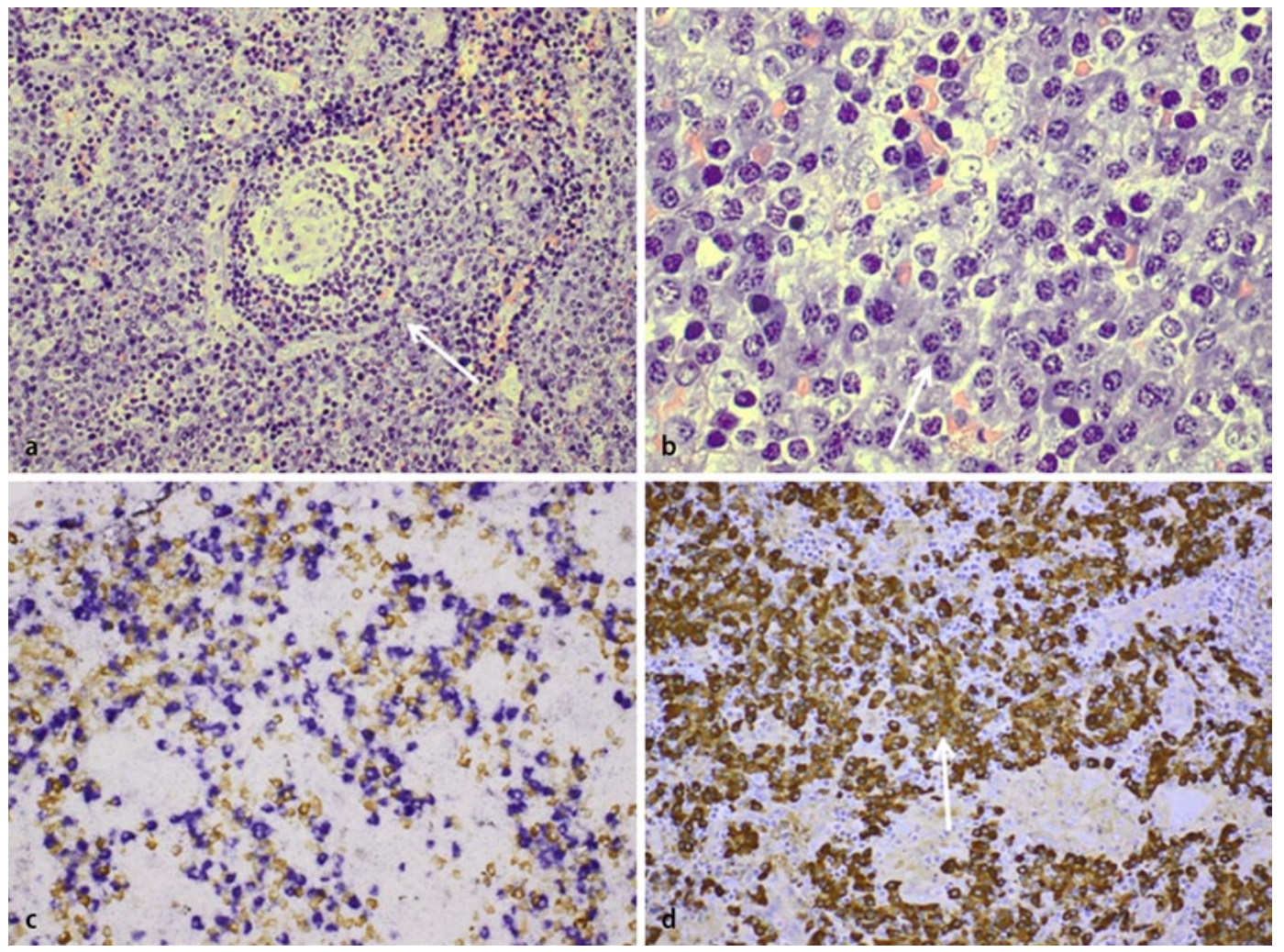

Abb. $2<$ a Übersichtsbild mit kleinem sekundärem Lymphfollikel, der von einer hohen Zahl an Plasmazellen umhüllt ist (Pfeil; Giemsa-Färbung, Vergr. 40:1). b Detail des plasmazellulären Infiltrats (Pfeil; GiemsaFärbung, Vergr. 60:1). c Die Doppelfärbung für leichte Ketten vom Typ к (braun) und Typ $\lambda$ (blau) beweist die polytypische Verteilung der Plasmazellen (Vergr. 40:1). d Immunhistochemie für lgG. Alle Plasmazellen exprimieren schwere Ketten von IgG (Pfeil; Vergr. 40:1)

\section{Histologie des mediastinalen Lymphknotens}

Überwiegend regressiv veränderte Lymphfollikel mit interfollikulärer Plasmozytose (• Abb.2). Das Bild passte morphologisch und immunhistochemisch zu einer plasmazellulären Variante des Morbus Castleman. Eine immunhistochemische Färbung für das latente nukleäre Antigen (LNAl) des humanen Herpesvirus Typ 8 (HHV-8) fiel negativ aus.
Erweiterte Laboruntersuchung und Knochenmarkpunktion

Die erweiterte Laboruntersuchung erbrachte folgende Ergebnisse: Interleukin(IL)-6 36,2 mg/l (<3,4 mg/l); HIV-Serologie negativ. Gesamtprotein $90 \mathrm{~g} / \mathrm{l}$ (60$78 \mathrm{~g} / \mathrm{l})$, polyklonale Hypergammaglobulinämie mit kleinem monoklonalem Anteil (Paraprotein vom Typ IgGk $9 \mathrm{~g} / \mathrm{l}$ ); Albumin $29 \mathrm{~g} / \mathrm{l}(35-52 \mathrm{~g} / \mathrm{l})$. Serologie: Zyto-
megalievirus(CMV)-IgG positiv, CMVIgM negativ, Epstein-Barr-Virus(EBV)IgG und EBV-IgM negativ; eine HHV-8Serologie wurde nicht durchgeführt. Das Knochenmark zeigte reaktive Veränderungen ohne Plasmazellvermehrung und ohne Hinweis auf eine Infiltration durch einen Morbus Castleman. 


\section{Diagnose}

\section{- Multizentrischer Morbus Castleman HIV- und HHV-8-negativ}

\section{Therapie}

Aufgrund des reduzierten Allgemeinzustands und der Pneumopathie entschieden wir uns gegen eine kombinierte Immunchemotherapie, z. B. mit Rituximab sowie Cyclophosphamid, Doxorubicin, Vincristin und Prednison (CHOP). Die initiale Therapie mit hoch dosiertem Methylprednisolon i.v. (30 mg/kg/Tag) mussten wir wegen subjektiver Unverträglichkeit abbrechen. Im Anschluss wurde die Patientin einer Behandlung mit Rituximab, einem monoklonalen Antikörper gegen CD20, unterzogen. Die Dosierung lag bei $375 \mathrm{mg} / \mathrm{m}^{2}$ wöchentlich für 4 Wochen. Darunter waren die mediastinale Lymphadenopathie und die Entzündungszeichen regredient, allerdings war die Wirkung nur vorübergehend, mussten wir doch bereits nach 7 Monaten ein symptomatisches Rezidiv diagnostizieren. Es folgten Therapien mit Thalidomid $50 \mathrm{mg} /$ Tag (kein Ansprechen, Arzneimittelexanthem), Etanercept (kein Ansprechen) und Anakinra (kein Ansprechen).

Ab Dezember 2007 erhielt die Patientin erstmals den IL-6-Rezeptor-Antagonisten Tocilizumab in einer Dosis von $8 \mathrm{mg} / \mathrm{kg}$ i.v., anschließend alle 14 Tage. Darunter kam es zu einer raschen und deutlichen klinischen und laborchemischen Besserung. Die Patientin wird nun seit mehr als 5 Jahren mit Tocilizumab behandelt. Die Therapie wird ohne Prämedikation verabreicht und bisher ohne kurzfristige und mittelfristige unerwünschte Wirkungen toleriert. Die Dosierung und das Dosierungsintervall veränderten wir nicht, da alle Versuche einer Verlängerung zu einer sofortigen symptomatischen Zunahme des Entzündungszustands führten. Der CRP-Wert beträgt seit Therapiebeginn meist $<25 \mathrm{mg} / \mathrm{l}$. IL-6 ist wegen des verzögerten Abbaus (kompetitive Besetzung des IL-6-Rezeptors durch Tocilizumab) konstant erhöht und liegt in einem Bereich von 300-600 mg/l (Norm: <3,4 mg/l; [10]). Das Hämoglobin ist normwertig. Auch die Lymphadeno- pathie und die interstitiellen Lungenveränderungen blieben stabil.

\section{Diskussion}

\section{Formen und klinisches Erscheinungsbild des Morbus Castleman}

Der Morbus Castleman ist eine seltene, primär nichtklonale lymphoproliferative Erkrankung von entdifferenzierten BLymphozyten und Plasmazellen, die erstmals 1956 von Dr. Benjamin Castleman beschrieben wurde [2]. Die Diagnosestellung erfolgt pathologisch-histologisch. Die wichtigsten histologischen Formen sind die plasmazelluläre, die hyalin-vaskuläre und die gemischte Variante. Neben der unizentrischen, histologisch fast immer hyalin-vaskulären Form, die mithilfe einer Lokaltherapie kurativ behandelbar ist, gibt es den multizentrischen Morbus Castleman ["multicentric Castleman disease“ (MCD)] mit ungünstiger Prognose. Die Erhebung des HIV- und HHV-8-Status bei Diagnosestellung ist entscheidend.

\section{$>$ Der Morbus Castleman ist bei} HIV-positiven Patienten wie das Kaposi-Sarkom mit HHV-8 assoziiert.

HHV-8 scheint ein wichtiger Auslöser der Erkrankung zu sein und birgt insbesondere das Potenzial der malignen Transformation bis hin zur Entwicklung hochmaligner, körperhöhlenassoziierter, plasmablastisch differenzierter Lymphome. HHV-8 wird in den Gefäßendothelzellen, den B-Lymphozyten sowie den Plasmablasten v. a. interfollikulär und in der Mantelzone nachgewiesen und produziert u. a. ein virales Homolog von IL-6 (vIL6; [11]).

Bei HIV-negativen Patienten mit MCD wird HHV-8 nur in etwa 40-50\% der Fälle nachgewiesen [12]. Es gibt klinische Hinweise, dass der HHV-8-assoziierte MCD ungeachtet des HIV-Status klinisch aber meist ähnlich verläuft: Die Patienten leiden unter Fieber und ausgeprägten konstitutionellen Symptomen, begleitet von einer starken Entzündungsreaktion. Typisch für Patienten mit HHV-8-Nachweis ist die Assoziation mit einer reaktiven Makrophagenaktivierung (hämo-
Internist 2013 · 54:1011-1015

DOI 10.1007/s00108-013-3327-3

๑) Springer-Verlag Berlin Heidelberg 2013

A. Wolfensberger $\cdot$ R. Benz $\cdot$ E.W. Russi $M$. Tinguely $\cdot$ C. Keller $\cdot$ B. Gerber Husten, Atemnot und B-Symptome bei einer 40-jährigen Frau

\section{Zusammenfassung}

Der Morbus Castleman ist eine seltene polyklonale, lymphoproliferative Erkrankung, bei der Mediatoren von Entzündungsreaktionen, $v$. a. Interleukin-6, eine wichtige pathophysiologische Rolle spielen. Zur Behandlung dieser Krankheit ist keine Standardtherapie etabliert. Wir berichten über den Fall einer 40-jährigen HIV-negativen Patientin mit primär pulmonaler Manifestation eines HHV8-negativen, plasmazellreichen multizentrischen Morbus Castleman. Verschiedene Therapieversuche mit Immunmodulatoren wurden durchgeführt, bevor eine Behandlung mit dem Interleukin-6-Rezeptor-Antikörper Tocilizumab begonnen wurde. Seit 5 Jahren ist der klinische Verlauf unter fortgesetzter Tocilizumabgabe stabil.

Schlüsselwörter

Morbus Castleman · Tocilizumab .

Interleukin-6 $\cdot$ Humanes

Immundefizienzvirus $\cdot$ Humanes

Herpesvirus Typ 8

\section{Dyspnoea, cough and $B$ symptoms in a 40-year-old woman}

\section{Abstract}

Multicentric Castleman's disease (MCD) is a rare polyclonal lymphoproliferative disorder that is typically accompanied by an overproduction of circulating cytokines (mainly interleukin-6). We here report the case of a 40-year-old HIV-negative woman with pulmonary manifestation of MCD. There is no standard treatment for MCD. In our patient, various treatment courses with immunomodulatory drugs were unsuccessful. Finally, treatment with the interleukin- 6 receptor antibody tocilizumab has resulted in continual clinical improvement over the last 5 years.

\section{Keywords}

Multi-centric Castleman's disease .

Tocilizumab - Interleukin- $6 \cdot$ Human immunodeficiency virus $\cdot$ Herpes virus 8 human 
phagozytische Lymphohistiozytose), mit Kaposi-Sarkomen (bis 50\% der Patienten) und mit einem erhöhten Risiko für die Entwicklung von Non-Hodgkin-Lymphomen [5].

Die Ursache des HHV-8-negativen MCD ist unklar. Bisher unbekannte virale Auslöser werden diskutiert. Die Beschwerden werden durch ein Zusammenspiel von multiplen, teilweise deregulierten Entzündungsmediatoren, v. a. IL-6, ausgelöst. IL-6 wird hier hauptsächlich in den follikulären dendritischen Zellen überproduziert und führt zur lymphovaskulären Proliferation sowie zu einer symptomatischen systemischen Entzündungsreaktion $[8,15]$.

Klinisch äußert sich der MCD im Allgemeinen mit B-Symptomatik, Hepatosplenomegalie und Lymphadenopathie. Im Labor zeigen sich

- quantitative Blutbildveränderungen:

- Anämie,

- Thrombozytose,

- Thrombopenie und

- Neutrophilie;

- eine Hypalbuminämie;

- eine polyklonale Hypergammaglobulinämie und

— erhöhte Entzündungszeichen.

Es besteht eine Überlappung des HHV8-negativen MCD mit dem POEMS-Syndrom (Polyneuropathie, Organomegalie, Endokrinopathie, monoklonale Gammopathie, Hautveränderungen; [4]).

Differenzialdiagnostisch muss ein MCD von

- malignen Lymphomen,

- einer Sarkoidose,

- chronischen Infekten,

- Immundefekten und

- IgG4-assoziierten autoimmun bedingten Erkrankungen

abgegrenzt werden.

Die Diagnose eines MCD bei unserer Patientin konnte durch die mediastinoskopisch durchgeführte Lymphknotenbiopsie gesichert werden. Der Nachweis eines Knochenmarkbefalls ist für die Diagnose eines MCD nicht notwendig. Der Zusammenhang zwischen MCD und interstitieller Pneumopathie ist bekannt und beruht neben der direkten Infiltration durch den MCD wahrscheinlich auch auf den durch Entzündungsmediatoren vermittelten Effekten.

\section{Therapieansätze}

Die Behandlung des MCD ist nicht standardisiert und beruht wegen der Seltenheit der Erkrankung auf Einzelfallberichten und kleineren Fallserien [3]. In Studien am besten untersucht ist die Therapie von HIV- und HHV-8-positiven MCD-Patienten mit dem Topoisomerasehemmer Etoposid und/oder Rituximab, einem Anti-CD20-Antikörper [1, 6, 7]. Die Rationale der Verwendung von Rituximab besteht darin, die HHV-8-infizierten, schwach CD20-positiven Plasmablasten und die meist CD20-positiven B-Lymphozyten zu eliminieren. Reife Plasmazellen exprimieren CD20 hingegen nicht. Obwohl der Beginn einer antiretroviralen Therapie grundsätzlich empfohlen wird, ist der Nutzen bei MCD im Gegensatz zur Behandlung des Kaposi-Sarkoms weniger klar. Die Wirkung einer gegen Herpesviren gerichteten Therapie wurde in vitro gezeigt. Zuverlässige In-vivo-Daten bei MCD fehlen aber. Kombinationschemotherapien sind für HIV-positive Patienten aufgrund der Toxizität meist nicht die Behandlung der ersten Wahl.

\section{》) Die Behandlung des Morbus Castleman beruht auf Einzelfallberichten und kleineren Fallserien}

Für HIV- und HHV-8-negative Patienten mit MCD ist die Gabe von Tocilizumab, einem Anti-IL-6-Rezeptor-Antikörper, prospektiv an einer japanischen Kohorte mit MCD untersucht worden [9]. Die inhibierende Wirkung auf die Entzündungsreaktion tritt rasch ein, hält aber nur an, solange das Medikament gegeben wird. In klinischer Erprobung ist seit Neuerem auch ein Anti-IL-6-Antikörper (Siltuximab; [13]). Obwohl nicht in klinischen Studien untersucht, besteht die Basistherapie meist in der Gabe von systemischen Kortikosteroiden. Verschiedene Zytostatika, z. B. Chlorambucil, Cyclophosphamid, Vincristin, Bleomycin, Doxorubicin, Etoposid und Vinblastin, wurden als Monotherapie, Kombinationsche- motherapie (z. B. CHOP) und/oder in Kombination mit Rituximab zur Behandlung des MCD eingesetzt [14]. Fallberichte und Fallserien weisen auf das Erreichen einer vorübergehenden partiellen oder kompletten Remission und seltener auch einer anhaltenden Remission hin. Die Remissionskriterien für den MCD sind allerdings nicht standardisiert.

Verschiedene immunmodulatorische und entzündungshemmende Medikamente und auch Substanzen, die wir aus der Myelombehandlung kennen, wurden zur Behandlung HIV-positiver und HIVnegativer Patienten mit MCD verwendet und sind teilweise in Fallberichten publiziert (Interferon- $\alpha$, IL-1-Rezeptor-Antagonist, Tumor-Nekrose-Faktor- $\alpha$-Blocker, Thalidomid, Bortezomib). Auch über Hochdosischemotherapien mit autologem Stammzellersatz wurde berichtet.

Unsere Patientin profitiert klinisch, laborchemisch und lungenfunktionell seit 5 Jahren ohne relevante Nebenwirkungen von einer fortgeführten Behandlung mit Tocilizumab. Im Falle eines Therapieversagens würden wir die Möglichkeit einer kombinierten Immunchemotherapie in Erwägung ziehen.

\section{Fazit für die Praxis}

- Ein systemischer Entzündungszustand mit Lymphadenopathie kann durch einen multizentrischen Morbus Castleman (MCD) bedingt sein.

- Entscheidend für die Diagnose ist die Gewinnung einer ausreichenden Menge Gewebe zur histologischen Untersuchung.

- Die Krankheit ist häufig mit HIV- und HHV-8-Infektionen assoziiert.

- Die Behandlung des MCD ist nicht standardisiert und sollte von einem erfahrenen, interdisziplinären Team durchgeführt werden.

- Monoklonale Antikörper, v. a. Tocilizumab und Rituximab, haben die therapeutischen Möglichkeiten wesentlich verbessert. 


\section{Korrespondenzadresse}

\section{Dr. B. Gerber}

Klinik für Hämatologie,

UniversitätsSpital Zürich

Rämistr. 100,

8091 Zürich

Schweiz

bernhard.gerber@usz.ch

\section{Einhaltung ethischer \\ Richtlinien}

Interessenkonflikt. B. Gerber, A. Wolfensberger, R. Benz, E.W. Russi, M. Tinguely und C. Keller geben an, dass kein Interessenkonflikt besteht.

Dieser Beitrag beinhaltet keine Studien an Menschen oder Tieren.

\section{Literatur}

1. Bower M (2010) How I treat HIV-associated multicentric Castleman disease. Blood 116:4415-4421

2. Castleman B, Iverson L, Menendez VP (1956) Localized mediastinal lymphnode hyperplasia resembling thymoma. Cancer 9:822-830

3. Dispenzieri A (2008) Castleman disease. Cancer Treat Res 142:293-330

4. Dispenzieri A, Armitage JO, Loe MJ et al (2012) The clinical spectrum of Castleman's disease. Am J Hematol 87:997-1002

5. Dossier A, Meignin V, Fieschi C et al (2013) Human herpesvirus 8-related Castleman disease in the absence of HIV infection. Clin Infect Dis 56:833-842

6. Gerard L, Berezne A, Galicier L et al (2007) Prospective study of rituximab in chemotherapy-dependent human immunodeficiency virus associated multicentric Castleman's disease: ANRS 117 CastlemaB Trial. J Clin Oncol 25:3350-3356

7. Hoffmann C, Schmid H, Muller M et al (2011) Improved outcome with rituximab in patients with HIV-associated multicentric Castleman disease. Blood 118:3499-3503

8. Leger-Ravet MB, Peuchmaur M, Devergne 0 et al (1991) Interleukin-6 gene expression in Castleman's disease. Blood 78:2923-2930

9. Nishimoto N, Kanakura Y, Aozasa K et al (2005) Humanized anti-interleukin- 6 receptor antibody treatment of multicentric Castleman disease. Blood 106:2627-2632

10. Nishimoto N, Terao K, Mima T et al (2008) Mechanisms and pathologic significances in increase in serum interleukin-6 (IL-6) and soluble IL-6 receptor after administration of an anti-IL- 6 receptor antibody, tocilizumab, in patients with rheumatoid arthritis and Castleman disease. Blood 112:39593964

11. Schulte KM, Talat N (2010) Castleman's disease - a two compartment model of HHV8 infection. Nat Rev Clin Oncol 7:533-543

12. Soulier J, Grollet L, Oksenhendler E et al (1995) Kaposi's sarcoma-associated herpesvirus-like DNAsequences in multicentric Castlemans disease. Blood 86:1276-1280
13. Van Rhee F, Fayad L, Voorhees P et al (2010) Siltuximab, a novel anti-interleukin-6 monoclonal antibody, for Castleman's disease. J Clin Oncol 28:3701-3708

14. Van Rhee F, Stone K, Szmania S et al (2010) Castleman disease in the 21st century: an update on diagnosis, assessment, and therapy. Clin Adv Hematol Oncol 8:486-498

15. Yoshizaki K, Matsuda T, Nishimoto N et al (1989) Pathogenic significance of interleukin-6 (IL-6/BSF2) in Castleman's disease. Blood 74:1360-1367

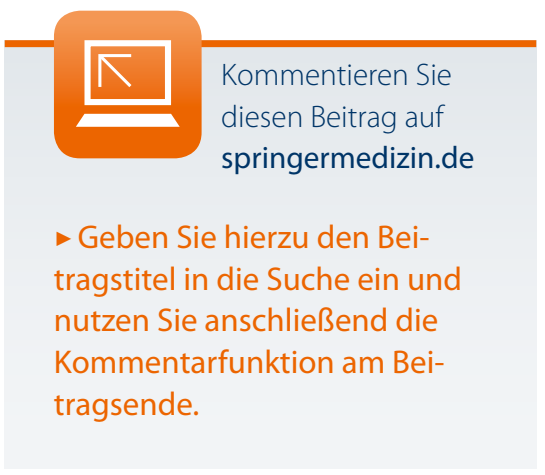

$\checkmark$ Geben Sie hierzu den Beitragstitel in die Suche ein und nutzen Sie anschließend die Kommentarfunktion am Beitragsende.

\section{Ausschreibung Christian-Lauritzen-Preis 2013}

Zur Prämierung hervorragender wissenschaftlicher Originalarbeiten stellt die Deutsche Menopause Gesellschaft e.V. aus Förderkreismitteln jährlich einen Gesamtbeitrag von Euro 5.000 zur Verfügung. Preiswürdige Arbeiten müssen neue Ergebnisse klinischer Forschung darstellen, die sich primär mit der sexualhormonabhängigen Gesundheit von Frauen und Männern in der zweiten Lebenshälfte auseinandersetzt. Eingeschlossen sind auch experimentelle Arbeiten, die eine unmittelbare Beziehung zu dieser Fragestellung herstellen.

Einzureichende Unterlagen:

Das Manuskript (maximal 30 Seiten DIN-A-4 inklusive Abbildungen, Tabellen und Referenzen; insgesamt 7 Kopien, deutsch oder englisch), das den Autorenrichtlinien einer im Jahr der Einreichung im Index Medicus gelisteten Zeitschrift entspricht, ist an den Präsidenten der Deutschen Menopause Gesellschaft e.V. zu schicken:

Prof. Dr. Alfred O. Mueck, Universitäts-Frauenklinik; Schwerpunkt für Endokrinologie und Menopause; Leiter Landesinstitut für Frauengesundheit Baden-Württemberg, Calwer Str.7, 72076 Tübingen

Bewerbungsfrist ist der 30 . September 2013

Quelle: Deutsche Menopause Gesellschafte.V. 